\title{
Almost periodic and asymptotically almost periodic functions: part I
}

\author{
Dhaou Lassoued ${ }^{1,2}$, Rahim Shah ${ }^{3}$ and Tongxing Li, ${ }^{4,5 *}$
}

\begin{tabular}{l}
\hline${ }^{*}$ Correspondence: \\
litongx2007@163.com \\
${ }^{4}$ LinDa Institute of Shandong \\
Provincial Key Laboratory of \\
Network Based Intelligent \\
Computing, Linyi University, Linyi, \\
P.R. China \\
${ }^{5}$ School of Information Science and \\
Engineering, Linyi University, Linyi, \\
P.R. China \\
Full list of author information is \\
available at the end of the article
\end{tabular}

available at the end of the article

\begin{abstract}
In this paper, we review indispensable properties and characterizations of almost periodic functions and asymptotically almost periodic functions in Banach spaces. Special accent is put on the Stepanov generalizations of almost periodic functions and asymptotically almost periodic functions. We also recollect some basic results regarding equi-Weyl-almost periodic functions and Weyl-almost periodic functions. The class of asymptotically Weyl-almost periodic functions, introduced in this work, seems to be not considered elsewhere even in the scalar-valued case. We actually introduce eight new classes of asymptotically almost periodic functions and analyze relations between them. In order to make a picture as complete and clear as possible, several illustrating examples and counter-examples are given. It is worth noting that the topics dealt with in this paper seem to be of an intrinsic connection with the problem of existence and uniqueness of solutions of differential and difference equations, in both determinist and stochastic cases.
\end{abstract}

MSC: $42 \mathrm{~A} 75$

Keywords: almost periodic function; asymptotically almost periodic function

\section{Springer}

\section{Introduction}

The theory of almost periodic functions has gradually been increased to a comprehensive and extensive theory by the contributions of numerous mathematicians. Indeed, the prehistory of almost periodicity begins with Esclangon and Bohl. The theory of almost periodic functions was developed in its main features by Bohr as a generalization of pure periodicity in three rather long papers [1-3], under the common title 'Zur Theorie der fastperiodischen Funktionen' in 1925 and 1926. The first of these papers dealt with the almost periodic functions of a real variable, while the third one took up the case of a complex variable. Afterwards, the theory of almost periodic functions was continuously getting established by several mathematicians like Amerio and Prouse [4], Levitan [5], Besicovitch, Bochner, von Neumann, Fréchet, Pontryagin, Lusternik, Stepanov, Weyl, etc.; with respect to this matter, we cite [6-10] and the references therein. In 1962, Bochner [11] defined and studied the almost periodic functions with values in Banach spaces. He showed that these functions include certain earlier generalizations of the notion of almost periodic functions. Some extensions of Bohr's concept have been introduced, most notably by Besicovitch, Stepanov, Weyl and Eberlein. One can remark that speaking about Stepanov,

(c) The Author(s) 2018. This article is distributed under the terms of the Creative Commons Attribution 4.0 International License (http://creativecommons.org/licenses/by/4.0/), which permits unrestricted use, distribution, and reproduction in any medium, provided you give appropriate credit to the original author(s) and the source, provide a link to the Creative Commons license, and indicate if changes were made. 
Weyl or Besicovitch metrics implicitly means dealing with the related quotient spaces, because otherwise we should rather speak about Stepanov, Weyl or Besicovitch.

In fact, the first motivation for the study of almost periodic functions is the set of various ways to combine periodic functions with different periods. For instance, the function $x \mapsto \cos x+\cos (5 x)$ is periodic, and this remains true when 5 is replaced by any other rational number. However, the sum of the periodic functions $x \mapsto e^{i x}$ and $x \mapsto e^{i \sqrt{2} x}$ is not periodic. Hence, when such functions, obtained by using a combination of periodic functions, are not periodic, they are not without properties: they are almost periodic functions. In the courses of mechanics, we usually encounter some two-dimensional differential systems of the form $x^{\prime}=A x+e(t)$, where $A$ is a $2 \times 2$ matrix with purely imaginary eigenvalue and $e(\cdot)$ is a periodic exterior force. It is well known that when these forced systems possess a periodic oscillation, then the period of this oscillation is exactly the period of the exterior force. It is not mentioned in these courses, but these forced systems possess almost periodic solutions. More generally, we know that when all the solutions of an autonomous linear finite dimensional system are bounded, then all these solutions are almost periodic. In more physical terms, the almost periodic trajectories are trajectories with a discrete spectrum. Besides, among the actual literature about the chaos theory, a famous model of transition towards the chaos is the Landau-Hopf model [12] where the involved potential is an almost periodic potential. Maurice Allais (Nobel Price of Economics) has written a wide work about the foundations of the theory of probabilities [13]. The major conclusion of his work is the following: many natural phenomena are considered as stochastic phenomena, but, in fact, they are almost periodic phenomena which are badly understood. In support of his viewpoint, Allais has established (with rigorous proofs [13]) a mathematical theorem which says that the samplings of an almost periodic function converge to the Laplace-Gauss distribution.

Ever since their introduction by Bohr in the mid-twenties, almost-periodic (a.p.) functions have played an important role in various branches of mathematics. Also, in the course of time, various variants and extensions of Bohr's concept have been introduced, most notably by Besicovitch, Stepanov and Weyl. Accordingly, there are a number of monographs and papers covering a wide spectrum of notions of almost periodicity and applications (see, for instance, the large list of references [14, Chapters 1 and 2]). An extension of Bohr's original (scalar) concept of a different kind is the generalization to vectorvalued almost-periodic functions, starting with Bochner's work in the thirties. Here, too, are a number of monographs on the subject, most notably by Amerio and Prouse [4] and Levitan and Zhikov [15]. This vector-valued (Banach space-valued) case is particularly important for applications to (the asymptotic behavior of solutions to) differential equations and dynamical systems.

As aforementioned, the notion and properties of almost periodic functions, either in their initial or in generalized form, turned out to be of great importance in various fields of analysis, function theory, topology and applied mathematics. The necessity of a manuscript giving a concise and systematic exposition of the fundamentals of the theory of almost periodic functions was becoming more and more obvious. The task of writing such a manuscript in a just only one part was an arduous one. Therefore, it is not astonishing that the present article will lead to other future works running in the same aim.

In the present article, we study the basic properties of almost periodic functions and asymptotically almost periodic functions. These topics are intrinsically connected with 
the problem of existence and uniqueness of solutions of differential equations. To give a complete and clear picture for the different spaces studied in our work, we illustrate them by several examples and counter-examples.

Throughout this paper, we use the usual notation $\mathbb{N}, \mathbb{Z}, \mathbb{R}$ and $\mathbb{C}$ for the sets of natural, integer, real and complex numbers, respectively.

For any real number $s \in \mathbb{R}$, we denote $\lfloor s\rfloor=\sup \{l \in \mathbb{Z}: s \geq l\}$ and $\lceil s\rceil=\inf \{l \in \mathbb{Z}: s \leq l\}$.

Unless stated otherwise, we assume that $X$ is an infinite-dimensional complex Banach space, and the norm of an element $x \in X$ is denoted by $\|x\|$. Assuming that $Y$ is another complex Banach space, we denote by $L(X, Y)$ the space consisting of all continuous linear mappings from $X$ into $Y$ and $L(X) \equiv L(X, X)$. The norm on $L(X, Y)$ shall be denoted by the same notation $\|\cdot\|$. The topology on $L(X, Y)$ and $X^{*}:=L(X, \mathbb{C})$, the dual space of $X$, are introduced in the usual way. The symbol $I$ denotes the identity operator on $X$. In some places, we need to have two different pivot spaces, thus we sometimes use the symbols $Y, Z, \ldots, E$ in place of $X$.

Let $I=\mathbb{R}$ or $I=[0, \infty)$. The space of all Bochner integrable functions from $I$ into $X$ is denoted by $L^{1}(I: X)$, equipped with the norm $\|f\|_{1}=\int_{I}\|f(t)\| d t$. For $1 \leq p<\infty$ and $(\Omega, \mathcal{R}, \mu)$ a measure space, by $L^{p}(\Omega: X)$ we denote the space consisting of all strongly $\mu$ measurable functions $f: \Omega \rightarrow X$ such that $\|f\|_{p}:=\left(\int_{\Omega}\|f(\cdot)\|^{p} d \mu\right)^{1 / p}$ is finite. By $C_{b}(I: X)$ we denote the space consisting of all bounded continuous functions from $I$ into $X$. The symbol $C_{0}([0, \infty): X)$ denotes the closed subspace of $C_{b}(I: X)$ consisting of functions vanishing as the module of the argument tends to infinity. By $\mathrm{BUC}(I: X)$ we denote the space consisting of all bounded uniformly continuous functions from $I$ to $X$. The sup-norm turns these spaces into Banach's. The notation $c_{0}$ will be deserved to the space of all complex sequences $\left(a_{n}\right)_{n}$ that converge to zero at infinity, that is, such that $\lim _{n \rightarrow \infty}\left|a_{n}\right|=0$. Sometimes, we use the notation $X^{I}$ for the set of all applications from $I$ into $X$. For an application $f \in X^{I}, R(f)$ denotes its range (or its image).

Our paper is organized in three big sections. In the first section, we study general almost periodic functions and asymptotically almost periodic ones. In the second section, we deal with the Stepanov generalization for almost periodicity and asymptotic almost periodicity. Then, Weyl almost periodic functions and asymptotically almost periodic functions are considered. These sections are mutually closely connected since we analyze some comparison relations linking the different functional spaces defined in each paragraph.

\section{Almost periodic functions and asymptotically almost periodic functions}

As underlined above, the concept of almost periodicity was introduced by Danish mathematician Bohr around 1924-1926 and later generalized by many other authors (cf. [10, 15-20] for more details on the subject).

Let $I=\mathbb{R}$ or $I=[0, \infty)$, and let $f: I \rightarrow \mathbb{R}$ be a continuous function. Given $\varepsilon>0$, we call $\tau>0$ an $\varepsilon$-period for $f(\cdot)$ if and only if, for all $t \in I$,

$$
\|f(t+\tau)-f(t)\| \leq \varepsilon
$$

The set consisting of all $\varepsilon$-periods for $f(\cdot)$ is denoted by $\mathcal{V}(f, \varepsilon)$.

It is said that the function $f(\cdot)$ is almost periodic, a.p. for short, if and only if, for each $\varepsilon>0$, the set $\mathcal{V}(f, \varepsilon)$ is relatively dense in $I$, which means that there exists a constant $l>0$ such that any subinterval of $I$ of length $l$ meets $\mathcal{V}(f, \varepsilon)$. 
Since for each $\varepsilon>0$ we have $\mathcal{V}(f, \varepsilon) \subset \mathcal{V}(\|f\|, \varepsilon)$, which is a consequence of the inequality \|\|$x\|-\| y\|\mid \leq\| x-y \|, x, y \in X$, it immediately follows from the definition that the almost periodicity of function $f: I \rightarrow X$ implies the almost periodicity of scalar-valued function $\|f\|: I \rightarrow \mathbb{R}$. Furthermore, it can be easily seen that the almost periodicity of function $f: I \rightarrow X$ implies the almost periodicity of vector-valued functions $f(\cdot+a)$ and $f(a \cdot)$, where $a \in I$.

We call $f(\cdot)$ weakly almost periodic, w.a.p. for short, if and only if for each $x^{*} \in X^{*}$ the function $x^{*}(f(\cdot))$ is almost periodic (it is well known that any function $f \in \operatorname{BUC}(I: X)$ which has a relatively compact range in $X$ and which is w.a.p., needs to be a.p., cf. [21, Proposition 4.5.12]). A family of functions $\mathcal{F} \subset X^{I}$ is said to be uniformly almost periodic if and only if, for each $\varepsilon>0$, there exists a constant $l>0$ such that any subinterval of $I$ of length $l$ contains a number $\tau>0$ such that (2.1) holds for all $f \in \mathcal{F}$.

The space consisting of all almost periodic functions from the interval $I$ into $X$ will be denoted by $\operatorname{AP}(I: X)$. Equipped with the sup-norm, $\operatorname{AP}(I: X)$ becomes a Banach space.

For the sequel, we need some preliminaries from the pioneering paper [22] by Bart and Goldberg. The translation semigroup $(W(t))_{t \geq 0}$ on $\mathrm{AP}([0, \infty): X)$, given by $[W(t) f](s):=$ $f(t+s), t \geq 0, s \geq 0, f \in \mathrm{AP}([0, \infty): X)$, consists solely of surjective isometries $W(t)(t \geq 0)$ and can be extended to a $C_{0}$-group $(W(t))_{t \in \mathbb{R}}$ of isometries on $\operatorname{AP}([0, \infty): X)$, where $W(-t):=W(t)^{-1}$ for $t>0$. Furthermore, the mapping $E: \operatorname{AP}([0, \infty): X) \rightarrow \operatorname{AP}(\mathbb{R}: X)$, defined by

$$
[E f](t):=[W(t) f](0), \quad t \in \mathbb{R}, f \in \operatorname{AP}([0, \infty): X),
$$

is a linear surjective isometry and $E f$ is the unique continuous almost periodic extension of a function $f$ from $\operatorname{AP}([0, \infty): X)$ to the whole real line. We have that $[E(B f)]=B(E f)$ for all $B \in L(X)$ and $f \in \operatorname{AP}([0, \infty): X)$.

The most intriguing properties of almost periodic vector-valued functions are collected in the following two theorems (in the case that $I=\mathbb{R}$ these assertions are well known in the existing literature, in the case that $I=[0, \infty)$, then these assertions can be deduced by using their validity in the case $I=\mathbb{R}$ and the properties of extension mapping $E(\cdot)$ ).

Theorem 2.1 Let $f \in \operatorname{AP}(I: X)$. Then the following assertions hold:

(1) $f \in \operatorname{BUC}(I: X)$;

(2) if $g \in \mathrm{AP}(I: X), h \in \mathrm{AP}(I: \mathbb{C}), \alpha, \beta \in \mathbb{C}$, then $\alpha f+\beta g$ and hf belong to $\operatorname{AP}(I: X)$;

(3) Bohr's transform of $f(\cdot)$

$$
P_{r}(f):=\lim _{t \rightarrow \infty} \frac{1}{t} \int_{0}^{t} e^{-i r s} f(s) d s
$$

exists for all $r \in \mathbb{R}$ and

$$
P_{r}(f)=\lim _{t \rightarrow \infty} \frac{1}{t} \int_{\alpha}^{t+\alpha} e^{-i r s} f(s) d s
$$

for all $\alpha \in I$ and $r \in \mathbb{R}$;

(4) if $P_{r}(f)=0$ for all $r \in \mathbb{R}$, then $f(t)=0$ for all $t \in I$;

(5) $\sigma(f):=\left\{r \in \mathbb{R}: P_{r}(f) \neq 0\right\}$ is at most countable; 
(6) if $c_{0} \nsubseteq X$, which means that $X$ does not contain an isomorphic copy of $c_{0}, I=\mathbb{R}$ and $g(t)=\int_{0}^{t} f(s) d s(t \in \mathbb{R})$ is bounded, then $g \in \operatorname{AP}(\mathbb{R}: X) ;$

(7) if $\left(g_{n}\right)_{n \in \mathbb{N}}$ is a sequence in $\mathrm{AP}(I: X)$ and $\left(g_{n}\right)_{n \in \mathbb{N}}$ converges uniformly to $g$, then $g \in \operatorname{AP}(I: X)$

(8) if $I=\mathbb{R}$ and $f^{\prime} \in \mathrm{BUC}(\mathbb{R}: X)$, then $f^{\prime} \in \mathrm{AP}(\mathbb{R}: X)$;

(9) (spectral synthesis) $f \in \overline{\operatorname{span}\left\{e^{i \mu} x: \mu \in \sigma(f), x \in R(f)\right\}}$;

(10) $R(f)$ is relatively compact in $X$;

(11) we have

$$
\|f\|_{\infty}=\sup _{t \geq t_{0}}\|f(t)\|, \quad t_{0} \in I
$$

(12) if $I=\mathbb{R}$ and $g \in L^{1}(\mathbb{R})$, then $g \star f \in \mathrm{AP}(\mathbb{R}: X)$, where $(g \star f)(t)=\int_{-\infty}^{\infty} g(t-s) f(s) d s$, $t \in \mathbb{R}$.

Theorem 2.2 (Bochner's criterion) Let $f \in \mathrm{BUC}(\mathbb{R}: X)$. Then $f(\cdot)$ is almost periodic if and only if, for any sequence $\left(b_{n}\right)_{n}$ of numbers from $\mathbb{R}$, there exists a subsequence $\left(a_{n}\right)_{n}$ of $\left(b_{n}\right)_{n}$ such that $\left(f\left(\cdot+a_{n}\right)\right)_{n}$ converges in $\operatorname{BUC}(R: X)$.

Remark 2.3 It is worth noting that assertion (8) in Theorem 2.1 holds in the case that $I=[0, \infty)$. More precisely, letting $f \in \operatorname{AP}([0, \infty): X)$ and $f^{\prime} \in \operatorname{BUC}([0, \infty): X)$, then $f^{\prime} \in$ $\operatorname{AP}([0, \infty): X)$. To see this, it suffices to apply assertion (7) from the same theorem by noticing that the sequence defined by $f_{n}(t):=n[f(t+1 / n)-f(t)], t \geq 0$ of almost periodic functions converges uniformly to $f(t)$ for $t \geq 0$, because

$$
\begin{aligned}
\left\|f_{n}(t)-f(t)\right\| & =\left\|n \int_{t}^{t+1 / n}\left[f^{\prime}(s)-f^{\prime}(t)\right] d s\right\| \\
& \leq n \int_{t}^{t+1 / n}\left\|f^{\prime}(s)-f^{\prime}(t)\right\| d s, \quad t \geq 0
\end{aligned}
$$

and $f^{\prime}(\cdot)$ is bounded uniformly continuous on $[0, \infty)$.

Before proceeding any further, we would like to mention that the necessary and sufficient condition for $X$ to contain $c_{0}$ is given in [21, Theorem 4.6.14] : $c_{0} \subseteq X$ if and only if there exists a divergent series $\sum_{n=1}^{\infty} x_{n}$ in $X$ which is unconditionally bounded, i.e., there exists a constant $M>0$ such that $\left\|\sum_{j=1}^{m} x_{n_{j}}\right\| \leq M$, whenever $n_{j} \in \mathbb{N}(j=1,2, \ldots, m)$ such that $n_{1}<n_{2}<\cdots<n_{m}$. The importance of condition $c_{0} \nsubseteq X$ has been recognized already by Bohr and later employed by many others (see, e.g., Kadet's theorem [21, Theorem 4.6.11]).

By either $\operatorname{AP}(\Lambda: X)$ or $\operatorname{AP}_{\Lambda}(I: X)$, where $\Lambda$ is a nonempty subset of $I$, we denote the vector subspace of $\mathrm{AP}(I: X)$ consisting of all functions $f \in \operatorname{AP}(I: X)$ for which the inclusion $\sigma(f) \subseteq \Lambda$ holds good. It can be easily seen that $\operatorname{AP}(\Lambda: X)$ is a closed subspace of $\operatorname{AP}(I: X)$ and therefore Banach space itself.

The relative compactness of subsets in $\operatorname{AP}(I: X)$ has been examined by Corduneanu [23] (see also [17, Theorem 3.11]). A function $f \in \mathrm{BUC}(I: X)$ is said to be weakly almost periodic in the sense of Eberlein if and only if $\{f(\cdot+s): s \in I\}$ is relatively weakly compact in $X$. This important class of functions will not be considered in the sequel (for further details concerning this intriguing topic and connections between almost periodicity and 
Carleman spectrum of functions, one may refer to the monograph [21] and the references cited therein).

\subsection{Asymptotically almost periodic functions}

The notion of an asymptotically almost periodic function was introduced by Fréchet in 1941 (for more details concerning the vector-valued asymptotically almost periodic functions and asymptotically almost periodic differential equations, see, e.g., [17, 18, 24-30]). A function $f \in C_{b}([0, \infty): X)$ is said to be asymptotically almost periodic if and only if, for every $\varepsilon>0$, we can find numbers $l>0$ and $M>0$ such that every subinterval of $[0, \infty)$ of length $l$ contains, at least, one number $\tau$ such that $\|f(t+\tau)-f(t)\| \leq \varepsilon$ for all $t \geq M$. The space consisting of all asymptotically almost periodic functions from $[0, \infty)$ into $X$ will be denoted by $\operatorname{AAP}([0, \infty): X)$.

It is well known that (see Ruess, Summers, and Vũ Quôc Phóng [27, 31-33]), for any function $f \in C([0, \infty): X)$, the following statements are equivalent:

(i) $f \in \operatorname{AAP}([0, \infty): X)$;

(ii) there exist uniquely determined functions $g \in \mathrm{AP}([0, \infty): X)$ and $\Phi \in C_{0}([0, \infty): X)$ such that $f=g+\Phi$;

(iii) the set $H(f):=\{f(\cdot+s): s>0\}$ is relatively compact in $C_{b}([0, \infty): X)$, which means that for any sequence $\left(b_{n}\right)_{n}$ of nonnegative real numbers there exists a subsequence $\left(a_{n}\right)_{n}$ of $\left(b_{n}\right)_{n}$ such that $\left(f\left(\cdot+a_{n}\right)\right)_{n}$ converges in $C_{b}([0, \infty): X)$.

The functions $g$ and $\Phi$ from (ii) are called the principal and corrective terms of the function $f$, respectively. Then we know that $\overline{R(g)} \subseteq \overline{R(f)}$ (see, e.g., [17, Lemma 3.43]).

By $C_{0}([0, \infty) \times Y: X)$, we denote the space of all continuous functions $h:[0, \infty) \times Y \rightarrow X$ such that $\lim _{t \rightarrow 0} h(t, y)=0$ uniformly for $y$ in any compact subset of $Y$. A continuous function $f: I \times Y \rightarrow X$ is called uniformly continuous on bounded sets, uniformly for $t \in I$ if and only if, for every $\varepsilon>0$ and every bounded subset $K$ of $Y$, there exists a number $\delta_{\varepsilon, K}>0$ such that $\|f(t, x)-f(t, y)\| \leq \varepsilon$ for all $t \in I$ and all $x, y \in K$ satisfying that $\|x-y\| \leq \delta_{\varepsilon, K}$. If $f: I \times Y \rightarrow X$, then we define $\hat{f}: I \times Y \rightarrow L^{p}([0,1]: X)$ by $f(\hat{t}, y):=f(t+\cdot, y), t \geq 0, y \in Y$. For the purpose of research of (asymptotically) almost periodic properties of solutions to semilinear Cauchy inclusions, we need to remind ourselves of the following well-known definitions and results (see, e.g., Zhang [34], Long and Ding [35] and Proposition 2.6 below).

Definition 2.4 Let $1 \leq p<\infty$.

(1) A function $f: I \times Y \rightarrow X$ is called almost periodic if and only if $f(\cdot, \cdot)$ is bounded, continuous as well as, for every $\varepsilon>0$ and every compact $K \subset Y$, there exists an $l(\varepsilon, K)>0$ such that every subinterval $J \subset I$ of length $l(\varepsilon, K)$ contains a number $\tau$ with the property that $\|f(t+\tau, y)-f(t, y)\| \leq \varepsilon$ for all $t \in I, y \in K$. The collection of such functions will be denoted by $\operatorname{AP}(I \times Y: X)$.

(2) A function $f:[0, \infty) \times Y \rightarrow X$ is said to be asymptotically almost periodic if and only if it is bounded continuous and admits a decomposition $f=g+q$, where $g \in \operatorname{AP}([0, \infty) \times Y: X)$ and $q \in C_{0}([0, \infty) \times Y: X)$. Denote by $\operatorname{AAP}([0, \infty) \times Y: X)$ the vector space consisting of all such functions.

The following composition principles are well known in the existing literature (see, e.g., [34]). 


\section{Theorem 2.5}

(1) Let $f \in \operatorname{AP}(I \times Y: X)$ and $h \in \operatorname{AP}(I: Y)$. Then the mapping $t \mapsto f(t, h(t)), t \in I$, belongs to the space $\mathrm{AP}(I: X)$.

(2) Let $f \in \operatorname{AAP}([0, \infty) \times Y: X)$ and $h \in \operatorname{AAP}([0, \infty): Y)$. Then the mapping $t \mapsto f(t, h(t)), t \geq 0$, belongs to the space $\operatorname{AAP}([0, \infty): X)$.

In Definition 2.4(2), a great number of authors assume a priori that $g \in \operatorname{AP}(\mathbb{R} \times Y: X)$. This is slightly redundant on account of the following proposition.

Proposition 2.6 Let $f:[0, \infty) \times Y \rightarrow X$ and let $S \subseteq Y$. Suppose that, for every $\varepsilon>0$, there exists an $l(\varepsilon, S)>0$ such that every subinterval $J \subseteq[0, \infty)$ of length $l(\varepsilon, S)$ contains a number $\tau$ with the property that $\|f(t+\tau, y)-f(t, y)\| \leq \varepsilon$ for all $t \geq 0, y \in S$ (this, in particular, holds provided that $f \in \operatorname{AP}(I \times Y: X))$.

Denote by $F(t, y)$ the unique almost periodic extension of function $f(t, y)$ from the interval $[0, \infty)$ to the whole real line for fixed $y \in S$.

Then, for every $\varepsilon>0$, with the same $l(\varepsilon, S)>0$ chosen as above, we have that every subinterval $J \subseteq \mathbb{R}$ of length $l(\varepsilon, S)$ contains a number $\tau$ with the property that $\| F(t+\tau, y)-$ $F(t, y) \| \leq \varepsilon$ for all $t \in \mathbb{R}, y \in S$.

Proof Let $\varepsilon>0$ be given in advance, $l(\varepsilon, S)>0$ be as above, and let $J=[a, b] \subseteq \mathbb{R}$. The assertion is clear provided that $a>0$. Suppose now that $a<0$. We choose a number $\tau_{0}>0$ arbitrarily. Then there exists a $\tau^{\prime} \in J=\left[\tau_{0}, \tau_{0}+b-a\right] \subseteq[0, \infty)$ such that $\| f\left(t+\tau_{0}, y\right)-$ $f(t, y) \| \leq \varepsilon$ for all $t \geq 0, y \in S$.

Since $\tau:=\tau^{\prime}-\tau_{0}-|a| \in J$, it suffices to show that $\|F(t+\tau, y)-F(t, y)\| \leq \varepsilon$ for all $t \in \mathbb{R}$, $y \in S$.

To this end, fix a number $t \in \mathbb{R}$ and an element $y \in S$. Since the mapping $s \mapsto F\left(s+\tau^{\prime}-\right.$ $\left.\tau_{0}-|a|, y\right)-F\left(s-\tau_{0}-|a|, y\right), s \in \mathbb{R}$ is almost periodic, equation (2.2) shows that

$$
\begin{aligned}
& \left\|F\left(s+\tau^{\prime}-\tau_{0}-|a|, y\right)-F\left(s-\tau_{0}-|a|, y\right)\right\| \\
& \quad \leq\left\|F\left(s+\tau^{\prime}-\tau_{0}-|a|, y\right)-F\left(s-\tau_{0}-|a|, y\right)\right\|_{\infty} \\
& =\sup _{s \geq \tau_{0}+|a|}\left\|F\left(s+\tau^{\prime}-\tau_{0}-|a|, y\right)-F\left(s-\tau_{0}-|a|, y\right)\right\| \\
& =\sup _{s \geq \tau_{0}+|a|}\left\|f\left(s+\tau^{\prime}-\tau_{0}-|a|, y\right)-f\left(s-\tau_{0}-|a|, y\right)\right\| \\
& =\sup _{s \geq 0}\left\|f\left(s+\tau^{\prime}, y\right)-f(s, y)\right\| \\
& \leq \varepsilon .
\end{aligned}
$$

This ends the proof of the proposition.

\section{Stepanov almost periodic functions and asymptotically Stepanov almost periodic functions}

Let $1 \leq p<\infty, l>0$, and $f, g \in L_{\mathrm{loc}}^{p}(I: X)$, where $I=\mathbb{R}$ or $I=[0, \infty)$.

We define the Stepanov 'metric' by

$$
D_{S_{l}}^{p}[f(\cdot), g(\cdot)]=\sup _{x \in I}\left[\frac{1}{l} \int_{x}^{x+l}\|f(t)-g(t)\|^{p} d t\right]^{1 / p} .
$$


Then we know that, for every two numbers $l_{1}, l_{2}>0$, there exist two positive real constants $k_{1}, k_{2}>0$ independent of $f, g$ such that

$$
k_{1} D_{S_{l_{1}}}^{p}[f(\cdot), g(\cdot)] \leq D_{S_{l_{2}}}^{p}[f(\cdot), g(\cdot)] \leq k_{2} D_{S_{l_{1}}}^{p}[f(\cdot), g(\cdot)]
$$

as well as that (see, e.g., [6, pp. 72-73]) in the scalar-valued case there exists

$$
D_{W}^{p}[f(\cdot), g(\cdot)]=\lim _{l \rightarrow \infty} D_{S_{l}}^{p}[f(\cdot), g(\cdot)]
$$

in $[0, \infty)$.

The distance appearing in (3.3) is called the Weyl distance of $f(\cdot)$ and $g(\cdot)$.

The Stepanov and Weyl 'norms' of $f(\cdot)$ are now respectively defined by

$$
\|f\|_{S_{l}^{p}}=D_{S_{l}}^{p}[f(\cdot), 0]
$$

and

$$
\|f\|_{W^{p}}=D_{W}^{p}[f(\cdot), 0]
$$

Taking into account (3.2), in the sequel of this section it will be appropriate to assume that $l_{1}=l_{2}=1$. We say that a function $f \in L_{\mathrm{loc}}^{p}(I: X)$ is Stepanov $p$-bounded, $S^{p}$-bounded shortly, if and only if

$$
\|f\|_{S^{p}}=\sup _{x \in I}\left[\int_{x}^{x+1}\|f(t)\|^{p} d t\right]^{1 / p}<\infty
$$

The space $L_{S}^{p}(I: X)$ consisting of all $S^{p}$-bounded functions becomes a Banach space when equipped with the above norm. A function $f \in L_{S}^{p}(I: X)$ is said to be Stepanov $p$-almost periodic, $S^{p}$-almost periodic shortly, if and only if the function $\hat{f}: I \rightarrow L^{p}([0,1]: X)$ defined by

$$
\hat{f}(t)(s)=f(t+s), \quad t \in I, s \in[0,1]
$$

is almost periodic (cf. [4] for more details).

\subsection{Asymptotically Stepanov almost periodic functions}

It is said that $f \in L_{S}^{p}([0, \infty): X)$ is asymptotically Stepanov $p$-almost periodic, asymptotically $S^{p}$-almost periodic shortly, if and only if $\hat{f}:[0, \infty) \rightarrow L^{p}([0,1]: X)$ is asymptotically almost periodic.

It is a well-known fact that if $f(\cdot)$ is an almost periodic (respectively, a.p.) function, then $f(\cdot)$ is also $S^{p}$-almost periodic (respectively, $S^{p}$-a.p.) for $1 \leq p<\infty$. The converse statement is false, however, as the following example from the book of Levitan [5] shows.

Example 3.1 Assume that $\alpha, \beta \in \mathbb{R}$ and $\alpha \beta^{-1}$ is a well-defined irrational number. Then the functions

$$
f(t)=\sin \frac{1}{2+\cos (\alpha t)+\cos (\beta t)}, \quad t \in \mathbb{R}
$$


and

$$
g(t)=\cos \frac{1}{2+\cos (\alpha t)+\cos (\beta t)}, \quad t \in \mathbb{R}
$$

are Stepanov $p$-almost periodic but not almost periodic $(1 \leq p<\infty)$.

Denote by $\operatorname{APS}^{p}(I: X)$ the space consisting of all $S^{p}$-almost periodic functions $f: I \rightarrow X$. For any $S^{p}$-almost periodic function $f(\cdot)$ and for any real number $\delta \in(0,1)$, we define the function

$$
f_{\delta}(t)=\frac{1}{\delta} \int_{t}^{t+\delta} f(s) d s, \quad t \in I
$$

Arguing as in the scalar-valued case [10], we can prove that the function $f_{\delta}(\cdot)$ is almost periodic $(0<\delta<1)$ as well as that $\left\|f_{\delta}-f\right\|_{S^{p}}$ converges to 0 as $\delta \rightarrow 0^{+}$.

Hereafter, we will also use the Bochner theorem, which asserts that any BUC function that is Stepanov $p$-almost periodic needs to be almost periodic $(1 \leq p<\infty)$.

The notion of a scalar $S^{p}$-almost periodic function, slightly different from the notion of usually considered weakly $S^{p}$-almost periodic function, is given as follows: a function $f \in$ $L_{S}^{p}(I: X)$ is said to be scalarly Stepanov $p$-almost periodic if and only if, for each $x^{*} \in X^{*}$, we have that the function $x^{*}(f):[0, \infty) \rightarrow \mathbb{C}$ defined by $x^{*}(f)(t):=x^{*}(f(t)), t \geq 0$, is Stepanov p-almost periodic.

Definition 3.2 A function $f: I \times Y \rightarrow X$ is called Stepanov $p$-almost periodic, $S^{p}$-almost periodic shortly, if and only if $\hat{f}: I \times Y \rightarrow L^{p}([0,1]: X)$ is almost periodic.

By [34, Theorem 2.6], we have that a bounded continuous function $f:[0, \infty) \times Y \rightarrow X$ is asymptotically almost periodic if and only if, for every $\varepsilon>0$ and every compact $K \subseteq Y$, there exist $l(\varepsilon, K)>0$ and $M(\varepsilon, K)>0$ such that every subinterval $J \subseteq[0, \infty)$ of length $l(\varepsilon, K)$ contains a number $\tau$ with the property that $\|f(t+\tau, y)-f(t, y)\| \leq \varepsilon$ for all $t>$ $M(\varepsilon, K), y \in K$.

We introduce the notion of an asymptotically Stepanov $p$-almost periodic function $f(\cdot, \cdot)$ as follows.

Definition 3.3 Let $1 \leq p<\infty$. A function $f:[0, \infty) \times Y \rightarrow X$ is said to be asymptotically $S^{p}$-almost periodic if and only if $\hat{f}:[0, \infty) \times Y \rightarrow L^{p}([0,1]: X)$ is asymptotically almost periodic. The collection of such functions will be denoted by $\operatorname{AAPS}^{p}([0, \infty) \times Y: X)$.

It is very elementary to prove that any asymptotically almost periodic function is also asymptotically Stepanov $p$-almost periodic $(1 \leq p<\infty)$.

We need the assertion of [36, Lemma 1].

Lemma 3.4 Suppose that $f:[0, \infty) \rightarrow X$ is an asymptotically $S^{p}$-almost periodic function. Then there are two locally $p$-integrable functions $g: \mathbb{R} \rightarrow X$ and $q:[0, \infty) \rightarrow X$ satisfying the following conditions:

(1) $g$ is $S^{p}$-almost periodic;

(2) $\hat{q}$ belongs to the class $C_{0}\left([0, \infty): L^{P}([0,1]: X)\right)$; 
(3) $f(t)=g(t)+q(t)$ for all $t \geq 0$.

Moreover, there exists an increasing sequence $\left(t_{n}\right)_{n \in \mathbb{N}}$ of positive reals such that $\lim _{n \rightarrow \infty} t_{n}=$ $\infty$ and $g(t)=\lim _{n \rightarrow \infty} f\left(t+t_{n}\right)$ a.e. $t \geq 0$.

Now we state the following two-variable analogue of Lemma 3.4.

Lemma 3.5 Suppose that $f:[0, \infty) \times Y \rightarrow X$ is an asymptotically $S^{p}$-almost periodic function. Then there are two functions $g: \mathbb{R} \times Y \rightarrow X$ and $q:[0, \infty) \times Y \rightarrow X$ satisfying that, for each $y \in Y$, the functions $g(\cdot, y)$ and $q(\cdot, y)$ are locally $p$-integrable as well as that the following hold:

(1) $\hat{g}: \mathbb{R} \times Y \rightarrow L^{p}([0,1]: X)$ is almost periodic;

(2) $\hat{q}$ belongs to the class $C_{0}\left([0, \infty) \times Y: L^{P}([0,1]: X)\right)$;

(3) $f(t, y)=g(t, y)+q(t, y)$ for all $t \geq 0$ and $y \in Y$.

Moreover, for every compact set $K \subseteq Y$, there exists an increasing sequence $\left(t_{n}\right)_{n \in \mathbb{N}}$ of positive reals such that $\lim _{n \rightarrow \infty} t_{n}=\infty$ and $g(t, y)=\lim _{n \rightarrow \infty} f\left(t+t_{n}, y\right)$ for all $y \in K$ and a.e. $t \geq 0$.

Proof By the foregoing, we have that $\hat{f}:[0, \infty) \times Y \rightarrow X$ is bounded continuous and admits a decomposition $\hat{f}=G+Q$, where $G \in \operatorname{AP}\left([0, \infty) \times Y: L^{p}([0,1]: X)\right)$ and $Q \in$ $C_{0}\left([0, \infty) \times Y: L^{p}([0,1]: X)\right)$. Moreover, the proof of [34, Theorem 2.6] shows that, for every compact set $K \subseteq Y$, there exists an increasing sequence $\left(t_{n}\right)_{n \in \mathbb{N}}$ of positive reals such that $\lim _{n \rightarrow \infty} t_{n}=\infty$ and $G(t, y)=\lim _{n \rightarrow \infty} \hat{f}\left(t+t_{n}, y\right)$ for all $y \in Y$ and $t \geq 0$. The remaining part of proof follows by applying Lemma 3.4 to the function $\hat{f}(\cdot, y)$ for a fixed element $y \in Y$ and the uniqueness of decomposition $g(\cdot)+q(\cdot)$ in this lemma.

In the case that the value of $p$ is irrelevant, we simply say that the function under our consideration is (asymptotically, scalarly) Stepanov almost periodic. Hereafter, we will use the following lemma (see, e.g., [6, p. 70] for the scalar-valued case).

Lemma 3.6 Let $-\infty<a<b<\infty, 1 \leq p^{\prime}<p^{\prime \prime}<\infty$, and $f \in L^{p^{\prime \prime}}([a, b]: X)$. Then $f \in$ $L^{p^{\prime}}([a, b]: X)$ and

$$
\left[\frac{1}{b-a} \int_{a}^{b}\|f(s)\|^{p^{\prime}} d s\right]^{1 / p^{\prime}} \leq\left[\frac{1}{b-a} \int_{a}^{b}\|f(s)\|^{p^{\prime \prime}} d s\right]^{1 / p^{\prime \prime}} .
$$

\section{Weyl almost periodic functions and asymptotically Weyl almost periodic functions}

Unless specified otherwise, in this section it will be always assumed that $I=\mathbb{R}$ or $I=[0, \infty)$. The pivot Banach space will be denoted by $X$. The notion of an (equi-)Weyl almost periodic function is given as follows (cf. also (3.1)).

Definition 4.1 Let $1 \leq p<\infty$ and $f \in L_{\mathrm{loc}}^{p}(I: X)$.

(1) We say that the function $f(\cdot)$ is equi-Weyl- $p$-almost periodic, $f \in e-W_{\text {ap }}^{p}(I: X)$ for short, if and only if, for each $\varepsilon>0$, we can find two real numbers $l>0$ and $L>0$ such that any interval $I^{\prime} \subseteq I$ of length $L$ contains a point $\tau \in I^{\prime}$ such that

$$
\sup _{x \in I} \frac{1}{l}\left[\int_{x}^{x+l}\|f(t+\tau)-f(t)\|^{p} d t\right]^{1 / p} \leq \varepsilon
$$


that is,

$$
D_{S_{l}}^{p}[f(\cdot+\tau), f(\cdot)] \leq \varepsilon
$$

(2) We say that the function $f(\cdot)$ is Weyl-p-almost periodic, $f \in W_{\mathrm{ap}}^{p}(I: X)$ for short, if and only if, for each $\varepsilon>0$, we can find a real number $L>0$ such that any interval $I^{\prime} \subseteq I$ of length $L$ contains a point $\tau \in I^{\prime}$ such that

$$
\lim _{l \rightarrow \infty} \sup _{x \in I} \frac{1}{l}\left[\int_{x}^{x+l}\|f(t+\tau)-f(t)\|^{p} d t\right]^{1 / p} \leq \varepsilon
$$

that is,

$$
\lim _{l \rightarrow \infty} D_{S_{l}}^{p}[f(\cdot+\tau), f(\cdot)] \leq \varepsilon
$$

Let us recall that

$$
\operatorname{APS}^{p}(I: X) \subseteq e-W_{\mathrm{ap}}^{p}(I: X) \subseteq W_{\mathrm{ap}}^{p}(I: X)
$$

in the set theoretical sense and that any of these two inclusions can be strict (see [37]).

For instance, the scalar-valued function $f: \mathbb{R} \rightarrow \mathbb{C}$ defined by $f(x):=\chi_{(0,1 / 2)}(x), x \in \mathbb{R}$ is not Stepanov 1-almost periodic, but it is equi-Weyl-almost-1-periodic (see, e.g., [37, Example 4.27]); and the scalar-valued function $f: \mathbb{R} \rightarrow \mathbb{C}$ defined by $f(x):=\chi_{(0, \infty)}(x), x \in \mathbb{R}$ is not equi-Weyl-almost-1-periodic, but it is Weyl-almost-1-periodic (see, e.g., [38, Example 1]). Here, $\chi(\cdot)$ denotes the characteristic function. We also want to point out that the space of scalar-valued functions $W_{\mathrm{ap}}^{p}(\mathbb{R}: \mathbb{R})$ seems to be defined and analyzed for the first time by Kovanko [39] in 1944 (according to the information given in the survey paper [37]).

It is well known that for any function $f \in L_{\mathrm{loc}}^{p}(I: X)$ its Stepanov boundedness is equivalent to its Weyl boundedness, i.e.,

$$
\|f\|_{S^{p}}<\infty \quad \Longleftrightarrow\|f\|_{W^{p}}<\infty .
$$

In the sequel, we use abbreviations $e-W_{\text {ap }}(I: X)$ and $W_{\text {ap }}(I: X)$ to denote the spaces $e-W_{\mathrm{ap}}^{1}(I: X)$ and $W_{\mathrm{ap}}^{1}(I: X)$, respectively (the case $p=1$ will be most important in our further analysis). Similarly, we say that a function is (equi)-Weyl-almost periodic if and only if it is (equi)-Weyl-1-almost periodic.

It is very important to state the following characteristic of the space $e-W_{\mathrm{ap}}^{p}(I: X)$, see, e.g., [37] for the scalar-valued case.

Theorem 4.2 Let $1 \leq p<\infty$ and $f \in L_{\mathrm{loc}}^{p}(I: X)$. Then $f \in e-W_{\mathrm{ap}}^{p}(I: X)$ if and only if, for every $\varepsilon>0$, there exists a trigonometric $X$-valued polynomial $P_{\varepsilon}(\cdot)$ such that

$$
D_{W}^{p}\left[P_{\varepsilon}(\cdot), f(\cdot)\right] \leq \varepsilon .
$$

A Bochner type theorem holds for Weyl almost periodic functions; see [5, 40]. 
Theorem 4.3 Let $1 \leq p<\infty$ and let $f \in W_{\mathrm{ap}}^{p}(I: X)$ be uniformly continuous. Then $f \in$ $\operatorname{AP}(I: X)$.

It is well known that the functions belonging to the space $e-W_{\mathrm{ap}}^{p}(I: X)$ need to be Weyl uniformly continuous in the following sense (see [6, p. 84]).

Theorem 4.4 Let $1 \leq p<\infty$ and $f \in W_{\mathrm{ap}}^{p}(I: X)$. Then, for every $\varepsilon>0$, there exist two finite numbers $L>0$ and $\delta_{0}>0$ such that

$$
D_{S_{L}}^{p}[f(\cdot+\delta), f(\cdot)] \leq \varepsilon
$$

for $|\delta| \leq \delta_{0}$.

For some other notions of Weyl-almost periodicity, like equi- $W^{p}$-normality and $W^{p}$ normality, we refer the reader to [37, Section 4].

\subsection{Asymptotically Weyl almost periodic functions}

For the beginning, we need to introduce the following notion. If $q \in L_{\mathrm{loc}}^{p}([0, \infty): X)$, then we define the function $q(\cdot, \cdot):[0, \infty) \times[0, \infty) \rightarrow X$ by

$$
q(t, s):=q(t+s), \quad t \geq 0, s \geq 0 .
$$

Definition 4.5 It is said that $q \in L_{\mathrm{loc}}^{p}([0, \infty): X)$ is Weyl-p-vanishing if and only if

$$
\lim _{t \rightarrow \infty}\|q(t, \cdot)\|_{W^{p}}=0, \quad \text { i.e., } \quad \lim _{t \rightarrow \infty} \lim _{l \rightarrow \infty} \sup _{x \geq 0}\left[\frac{1}{l} \int_{x}^{x+l}\|q(t+s)\|^{p} d s\right]^{1 / p}=0 .
$$

It is clear that, for any function $q \in L_{\mathrm{loc}}^{p}([0, \infty): X)$, we can replace the limits in (4.1). We say that $q \in L_{\mathrm{loc}}^{p}([0, \infty): X)$ is equi-Weyl- $p$-vanishing if and only if

$$
\lim _{l \rightarrow \infty} \lim _{t \rightarrow \infty} \sup _{x \geq 0}\left[\frac{1}{l} \int_{x}^{x+l}\|q(t+s)\|^{p} d s\right]^{1 / p}=0
$$

Since the second limit in (4.1) always exists in $[0, \infty)$ (on account of (3.3)) and the second limit in (4.2) always exists in $[0, \infty)$ (taking into account the fact that the mapping $t \mapsto \sup _{x \geq 0}\left[\int_{x}^{x+l}\|q(t+s)\|^{p} d s / l\right]^{1 / p}, t \geq 0$ is monotonically decreasing), condition (4.1) is equivalent to

$$
\begin{gathered}
\forall \varepsilon>0, \exists t_{0}(\varepsilon)>0, \forall t \geq t_{0}(\varepsilon), \exists l_{t}>0, \forall l>l_{t}: \\
\sup _{x \geq 0}\left[\frac{1}{l} \int_{x}^{x+l}\|q(t+s)\|^{p} d s\right]^{1 / p} \leq \varepsilon,
\end{gathered}
$$

while condition (4.2) is equivalent to

$$
\begin{gathered}
\forall \varepsilon>0, \exists l_{0}(\varepsilon)>0, \forall l \geq l_{0}(\varepsilon), \exists t_{l}>0, \forall t>t_{l}: \\
\sup _{x \geq 0}\left[\frac{1}{l} \int_{x}^{x+l}\|q(t+s)\|^{p} d s\right]^{1 / p} \leq \varepsilon .
\end{gathered}
$$


Now, assume that $q \in L^{p}([0, \infty): X)$. Then, for each $\varepsilon>0$, there exists a $t_{0}(\varepsilon)>0$ such that $\int_{t}^{\infty}\|q(s)\|^{p} d s \leq \varepsilon^{p}, t \geq t_{0}(\varepsilon)$. In particular, $\int_{t}^{t+1}\|q(s)\|^{p} d s \leq \varepsilon^{p}, t \geq t_{0}(\varepsilon)$, and the function $\hat{q}:[0, \infty) \rightarrow L^{p}([0,1]: X)$ belongs to the class $C_{0}\left([0, \infty): L^{p}([0,1]: X)\right)$. The converse statement is not true, however, since the scalar-valued function $q(t)=t^{-1 /(2 p)}, t>0$ satisfies that $\hat{q} \in C_{0}\left([0, \infty): L^{p}([0,1]: X)\right)$ and $q \notin L^{p}([0, \infty): X)$.

If $q \in L_{\mathrm{loc}}^{p}([0, \infty): X)$ and $\hat{q} \in C_{0}\left([0, \infty): L^{p}([0,1]: X)\right)$, then the computation

$$
\begin{aligned}
\sup _{x \geq 0}\left[\frac{1}{l} \int_{x}^{x+l}\|q(t+s)\|^{p} d s\right]^{1 / p} & \leq\left[\frac{1}{l}\left(\int_{x+t}^{x+t+1}+\cdots+\int_{x+t+\lceil l\rceil-1}^{x+t+\lceil l\rceil}\right)\|q(s)\|^{p} d s\right]^{1 / p} \\
& \leq\left(\frac{\varepsilon^{p}\lceil l\rceil}{l}\right)^{1 / p} \leq 2 \varepsilon
\end{aligned}
$$

holding for any $t \geq 0$, shows that the function $q(\cdot)$ is equi-Weyl- $p$-vanishing, with $l_{0}(\varepsilon)=1$ and $t_{l}=t_{0}(\varepsilon)$ chosen so that $\int_{t}^{t+1}\|q(s)\|^{p} d s \leq \varepsilon^{p}, t \geq t_{0}(\varepsilon)\left(l>l_{0}(\varepsilon)\right)$.

As the following simple counter-example shows, the converse statement does not hold in general.

\section{Example 4.6 Define}

$$
q(t):=\sum_{n=0}^{\infty} \chi_{\left[n^{2}, n^{2}+1\right]}(t), \quad t \geq 0
$$

Since $\int_{n^{2}}^{n^{2}+1}\|q(s)\|^{p} d s=1, n \in \mathbb{N}$, it is clear that $\hat{q} \notin C_{0}\left([0, \infty): L^{p}([0,1]: X)\right)$. On the other hand, the interval $[t, t+l]$ contains at most $\sqrt{t+l}-\sqrt{t}+2$ squares of nonnegative integers, so that

$$
\begin{aligned}
\frac{1}{l} \int_{x}^{x+l}\|q(t+s)\|^{p} d s & \leq \sup _{x \geq t} \frac{1}{l} \int_{t}^{t+l}\|q(s)\|^{p} d s \\
& \leq \frac{1}{l}(\sqrt{t+l}-\sqrt{t}+2) \leq \frac{1}{l}\left(2+\frac{l}{\sqrt{t}+\sqrt{l}}\right), \quad x \geq 0, t \geq 0,
\end{aligned}
$$

so that (4.4) holds with $l_{0}(\varepsilon)>0$ sufficiently large and $t_{l}=l\left(l>l_{0}(\varepsilon)\right)$.

If $q \in L_{\mathrm{loc}}^{p}([0, \infty): X)$ and $q(\cdot)$ is equi-Weyl-p-vanishing, then $q(\cdot)$ is Weyl-p-vanishing. To see this, assume that (4.4) holds with $l_{0}(\varepsilon)>0$ and put after that $t_{0}(\varepsilon):=t_{l_{0}}(\varepsilon)$. Therefore,

$$
\sup _{x \geq 0}\left[\frac{1}{l_{0}(\varepsilon)} \int_{x}^{x+l_{0}(\varepsilon)}\|q(t+s)\|^{p} d s\right]^{1 / p} \leq \varepsilon, \quad t \geq t_{l_{0}}(\varepsilon)
$$

For any fixed $t>t_{0}(\varepsilon)$, we set $l_{t}:=l_{0}(\varepsilon)$. Then it suffices to show that, for any $l>l_{t}$, we have

$$
\sup _{x \geq 0}\left[\frac{1}{l} \int_{x}^{x+l}\|q(t+s)\|^{p} d s\right]^{1 / p} \leq 2 \varepsilon
$$


This follows from (4.5) and a simple analysis involving the second inequality in part (i) of [38, Proposition 1]:

$$
\begin{aligned}
\sup _{x \geq 0}\left[\frac{1}{l} \int_{x}^{x+l}\|q(t+s)\|^{p} d s\right]^{1 / p} \\
\quad \leq 2^{1 / p} \sup _{x \geq 0}\left[\frac{1}{l_{0}(\varepsilon)} \int_{x}^{x+l_{0}(\varepsilon)}\|q(t+s)\|^{p} d s\right]^{1 / p}, \quad l>l_{t}=l_{0}(\varepsilon) .
\end{aligned}
$$

Again, the converse statement does not hold in general and a Weyl- $p$-vanishing function need not be equi-Weyl- $p$-vanishing.

Example 4.7 Define

$$
q(t):=\sum_{n=0}^{\infty} \sqrt{n} \chi_{\left[n^{2}, n^{2}+1\right]}(t), \quad t \geq 0 .
$$

Then it is clear that

$$
\begin{aligned}
\frac{1}{l} \int_{x}^{x+l}\|q(t+s)\|^{p} d s & \leq \sup _{x \geq t} \frac{1}{l} \int_{t}^{t+l}\|q(s)\|^{p} d s \\
& \leq \sqrt{\frac{t+l}{l^{2}}}, \quad x \geq 0, t \geq 0,
\end{aligned}
$$

so that (4.3) holds with $t_{0}(\varepsilon)>0$ chosen so that $\sqrt{1 /(t+l)} \leq \varepsilon^{p}$ and $l_{t}=t+l$. Hence, $q(\cdot)$ is Weyl- $p$-vanishing. On the other hand, $q(\cdot)$ cannot be equi-Weyl-p-vanishing because, for each number $l>1$, there does not exist a finite limit

$$
\lim _{l \rightarrow \infty}\left[\frac{1}{l} \int_{x}^{x+l}\|q(t+s)\|^{p} d s\right]^{1 / p} .
$$

To see this, it suffices to observe that, for each $t>0$ and $n \in \mathbb{N}$ such that $n \geq t^{2}$, we have

$$
\sup _{x \geq 0} \frac{1}{l} \int_{x}^{x+l}\|q(t+s)\|^{p} d s \geq \frac{\sqrt{n}}{l} \geq \frac{t}{l}
$$

Before proceeding further, we would like to note that an equi-Weyl- $p$-vanishing function $q(\cdot)$ need not be bounded as $t \rightarrow \infty$.

\section{Example 4.8 Define}

$$
q(t):=\sum_{n=0}^{\infty} n^{1 /(4 p)} \chi_{\left[n^{4}, n^{4}+1\right]}(t), \quad t \geq 0 .
$$

Then, similarly as in Example 4.6, we can prove that

$$
\begin{aligned}
\frac{1}{l} \int_{x}^{x+l}\|q(t+s)\|^{p} d s & \leq \sup _{x \geq t} \frac{1}{l} \int_{t}^{t+l}\|q(s)\|^{p} d s \\
& \leq \frac{1}{l}\left(2+\frac{l}{\sqrt{t}+\sqrt{l}}\right), \quad x \geq 0, t \geq 0,
\end{aligned}
$$

which yields the required conclusions. 
Denote by $W_{0}^{p}([0, \infty): X)$ and $e-W_{0}^{p}([0, \infty): X)$ the sets consisting of all Weyl- $p$ vanishing functions and equi-Weyl- $p$-vanishing functions, respectively. The symbol $S_{0}^{p}([0, \infty): X)$ will be used to denote the set of all functions $q \in L_{\mathrm{loc}}^{p}([0, \infty): X)$ such that $\hat{q} \in C_{0}\left([0, \infty): L^{p}([0,1]: X)\right)$.

By our previous considerations, Examples 4.6 and 4.7, we have the following result.

Theorem 4.9 The following inclusions hold:

$$
L^{p}([0, \infty): X) \subseteq S_{0}^{p}([0, \infty): X) \subseteq e-W_{0}^{p}([0, \infty): X) \subseteq W_{0}^{p}([0, \infty): X)
$$

and any of them can be strict.

Now, we introduce the following function spaces:

$$
\begin{aligned}
& \operatorname{AAPW}^{p}([0, \infty): X):=\operatorname{AP}([0, \infty): X)+W_{0}^{p}([0, \infty): X), \\
& e-\operatorname{AAPW}^{p}([0, \infty): X):=\operatorname{AP}([0, \infty): X)+e-W_{0}^{p}([0, \infty): X), \\
& \operatorname{AAPSW}^{p}([0, \infty): X):=\operatorname{APS}^{p}([0, \infty): X)+W_{0}^{p}([0, \infty): X), \\
& e-\operatorname{AAPSW}^{p}([0, \infty): X):=\operatorname{APS}^{p}([0, \infty): X)+e-W_{0}^{p}([0, \infty): X), \\
& e-W_{\text {aap }}^{p}([0, \infty): X):=e-W_{\mathrm{ap}}^{p}([0, \infty): X)+W_{0}^{p}([0, \infty): X), \\
& e e-W_{\text {aap }}^{p}([0, \infty): X):=e-W_{\mathrm{ap}}^{p}([0, \infty): X)+e-W_{0}^{p}([0, \infty): X), \\
& W_{\mathrm{aap}}^{p}([0, \infty): X):=W_{\mathrm{ap}}^{p}([0, \infty): X)+W_{0}^{p}([0, \infty): X), \\
& e-W_{\text {aap }}^{p}([0, \infty): X):=W_{\mathrm{ap}}^{p}([0, \infty): X)+e-W_{0}^{p}([0, \infty): X) .
\end{aligned}
$$

Then it is clear that

$$
\begin{aligned}
\operatorname{AAPW}^{p}([0, \infty): X) & \subseteq \operatorname{AAPSW}^{p}([0, \infty): X) \\
& \subseteq e-W_{\text {aap }}^{p}([0, \infty): X) \subseteq W_{\text {aap }}^{p}([0, \infty): X)
\end{aligned}
$$

and

$$
\begin{aligned}
e-\operatorname{AAPW}^{p}([0, \infty): X) & \subseteq e-\operatorname{AAPSW}^{p}([0, \infty): X) \\
& \subseteq e e-W_{\text {aap }}^{p}([0, \infty): X) \subseteq e-W_{\text {aap }}^{p}([0, \infty): X),
\end{aligned}
$$

and that any of these inclusions can be strict.

By the analysis contained in [37, Example 4.27], the function $f:[0, \infty) \rightarrow \mathbb{C}$ defined by $f(t):=\chi_{(0,1 / 2)}(t), t>0$ is equi-Weyl-almost periodic. Since this function is also in class $e-W_{0}^{p}([0, \infty): X)$, we have that the sums defining $e$ - $W_{\text {aap }}^{p}([0, \infty): X), e e-W_{\text {aap }}^{p}([0, \infty)$ : $X), W_{\text {aap }}^{p}([0, \infty): X)$ and $e-W_{\text {aap }}^{p}([0, \infty): X)$ are not direct. For the first four spaces $\operatorname{AAPW}^{p}([0, \infty): X), e-\operatorname{AAPW}^{p}([0, \infty): X), \operatorname{AAPSW}^{p}([0, \infty): X)$ and $e-\mathrm{AAPSW}^{p}([0, \infty):$ $X)$, the sums in their definitions are direct, which follow from the following proposition.

Proposition 4.10 Let $1 \leq p<\infty$. Then

$$
W_{0}^{p}([0, \infty): X) \cap \operatorname{APS}^{p}([0, \infty): X)=\{0\}
$$


Proof Assume $q \in W_{0}^{p}([0, \infty): X) \cap \operatorname{APS}^{p}([0, \infty): X)$. In order to prove that $q(t)=0$ for a.e. $t \geq 0$, it suffices to show that $\hat{q}(t)=0, t \geq 0$, in $L^{p}([0,1]: X)$. Since $\hat{q}(\cdot)$ is almost periodic, we only need to prove that any Bohr-Fourier coefficient of $\hat{q}(\cdot)$ is equal to zero, i.e., that

$$
\lim _{t \rightarrow \infty}\left(\int_{0}^{1}\left\|\frac{1}{t} \int_{0}^{t} e^{-i r s} q(s+v) d s\right\|^{p} d v\right)^{1 / p}=0, \quad r \in \mathbb{R} .
$$

To see that (4.6) holds good, observe first that

$$
\left(\int_{0}^{1}\left\|\frac{1}{t} \int_{0}^{t} e^{-i r s} q(s+v) d s\right\|^{p} d v\right)^{1 / p} \leq \frac{1}{t}\left(\int_{0}^{1}\left[\int_{0}^{t}\|q(s+v)\| d s\right]^{p} d v\right)^{1 / p}
$$

which can be further majorized by using Lemma 3.6:

$$
\leq \frac{1}{t}\left(\int_{0}^{1} t^{p-1} \int_{0}^{t}\|q(s+v)\|^{p} d s d v\right)^{1 / p}=t^{-1 / p}\left(\int_{0}^{1} \int_{0}^{t}\|q(s+v)\|^{p} d s d v\right)^{1 / p} .
$$

Hence, we need to prove that

$$
\lim _{t \rightarrow \infty} \frac{1}{t} \int_{0}^{1} \int_{0}^{t}\|q(s+v)\|^{p} d s d v=\lim _{t \rightarrow \infty} \frac{1}{t} \int_{s}^{s+t} \int_{0}^{t}\|q(r)\|^{p} d r d v=0
$$

Let $\varepsilon>0$ be given in advance. Since $q \in W_{0}^{p}([0, \infty): X)$, we know that there exist two finite numbers $t_{0}(\varepsilon)>0$ and $l_{0}(\varepsilon)>0$ such that, for every $l>l_{0}(\varepsilon)$,

$$
\sup _{x \geq 0}\left[\frac{1}{l} \int_{x}^{x+l}\left\|q\left(l_{0}(\varepsilon)+s\right)\right\|^{p} d s\right]^{1 / p} \leq \varepsilon
$$

Let $T_{0}(\varepsilon)>0$ be such that, for each $t>T_{0}(\varepsilon)$,

$$
t \geq t_{0}(\varepsilon)^{2} \text { and } \quad t-\sqrt{t} \geq l_{0}(\varepsilon)
$$

The validity of (4.9) clearly implies by (4.8) that

$$
\frac{1}{t-\sqrt{t}} \int_{s+\sqrt{t}}^{s+t}\|q(s)\|^{p} d s \leq \varepsilon, \quad s \in[0,1] .
$$

Since

$$
\begin{aligned}
\frac{1}{t} \int_{s}^{s+t}\|q(r)\|^{p} d r \leq & \frac{1}{t}\left(\int_{s}^{s+1}+\int_{s+1}^{s+2}+\cdots \int_{s+\lceil\sqrt{t}\rceil-1}^{s+\lceil\sqrt{t}\rceil}\right)\|q(r)\|^{p} d r \\
& +\frac{1}{t}\left(\int_{s+\lceil\sqrt{t}\rceil}^{s+\lceil\sqrt{t}\rceil+1}+\cdots+\int_{s+\lfloor t]}^{s+t}\right)\|q(r)\|^{p} d r \\
\leq & \frac{\lceil\sqrt{t}\rceil}{t}\|q\|_{S^{p}}+\frac{t-\lfloor t\rfloor}{t} \varepsilon,
\end{aligned}
$$

by $S^{p}$-boundedness of $q(\cdot)$ and (4.10), equation (4.7) holds true. The proof of the proposition is thereby complete. 
It is an easy task to prove that $W_{0}^{p}([0, \infty): X)$ and $e-W_{0}^{p}([0, \infty): X)$ are vector spaces, so that the introduced eight function spaces have a linear vector structure. Disregarding the term $([0, \infty): X)$ and taking into consideration the previously defined spaces AAP and $\mathrm{AAPS}^{p}$, we have the following inclusion diagram of asymptotically almost periodic function spaces (see Theorem 4.9):

\begin{tabular}{|c|c|c|c|c|}
\hline AAP & $\subseteq$ & $e-\mathrm{AAPW}^{p}$ & $\subseteq$ & $\mathrm{AAPW}^{p}$ \\
\hline In & & In & & $\ln$ \\
\hline \multirow[t]{5}{*}{$\mathrm{AAPS}^{p}$} & $\subseteq$ & $e$ - AAPSW $^{p}$ & $\subseteq$ & $\mathrm{AAPSW}^{p}$ \\
\hline & & In & & in \\
\hline & & $e e-W_{\text {aap }}^{p}$ & $\subseteq$ & $e-W_{\text {aap }}^{p}$ \\
\hline & & In & & In \\
\hline & & $e-W_{\text {aap }}^{p}$ & $\subseteq$ & $W_{\text {aap }}^{p}$ \\
\hline
\end{tabular}

By the foregoing, any inclusion of this diagram can be strict. Furthermore, for any two function spaces $A$ and $B$ belonging this diagram and satisfying additionally that there is no transitive sequence of inclusions connecting either $A$ and $B$ or $B$ and $A$, we have that $A \backslash B \neq \emptyset$ and $B \backslash A \neq \emptyset$ (the diagram can be expanded by constructing the sums of spaces of (equi-)Weyl almost periodic functions with $S_{0}^{p}([0, \infty): X)$, which will be not examined here).

We refer the reader to the paper [41] by Diagana et al. for more details about the notion of $S_{p}^{(n)}$-almost periodicity (the notion of $N$-almost periodicity is very well explored in the monograph [15] by Levitan and Zhikov. Stepanov cases have not been introduced so far, to the best knowledge of the authors). For an excellent survey of results about various classes of (Stepanov) almost periodic functions and (Stepanov) asymptotically almost periodic functions, we refer the reader to the review paper [37] by Andres et al. (cf. also Andres et al. [38]), already cited multiple times before.

We round off our paper by introducing the following important definition.

Definition 4.11 Let $I=\mathbb{R}$ or $I=[0, \infty),(R(t))_{t \in I} \subseteq L(X)$ be a strongly continuous operator family, and let $\bigoplus$ denote any of (asymptotically) almost periodic properties considered above. Then we say that $(R(t))_{t \in I}$ is $\bigoplus$ (asymptotically) almost periodic if and only if the mapping $t \mapsto R(t) x, t \in I$ is $\bigoplus$ (asymptotically) almost periodic for all $x \in X$. It is said that $(R(t))_{t \in I}$ is uniformly almost periodic if and only if the family $\{R(\cdot) x:\|x\| \leq 1\}$ is uniformly almost periodic.

\footnotetext{
Acknowledgements

The authors express their sincere gratitude to the editors and two anonymous referees for the careful reading of the original manuscript and useful comments that helped to improve the presentation of the results and accentuate important details. This research is supported by NNSF of P.R. China (Grant No. 61503171), CPSF (Grant No. 2015M582091), NSF of Shandong Province (Grant No. ZR2016JL021), DSRF of Linyi University (Grant No. LYDX2015BS001), and the AMEP of Linyi University, P.R. China. 


\section{Author details}

1 Département de Mathématiques, Faculté des Sciences de Gabès, Université de Gabès, Cité Erriadh, Tunisia. ${ }^{2}$ Laboratoire Équations aux dérivées partielles LEDP-LR03ES04, Faculté des Sciences de Tunis, Université de Tunis El Manar, Tunis, Tunisia. ${ }^{3}$ Department of Mathematics, University of Peshawar, Peshawar, Pakistan. ${ }^{4}$ LinDa Institute of Shandong Provincial Key Laboratory of Network Based Intelligent Computing, Linyi University, Linyi, P.R. China. ${ }^{5}$ School of Information Science and Engineering, Linyi University, Linyi, P.R. China.

\section{Publisher's Note}

Springer Nature remains neutral with regard to jurisdictional claims in published maps and institutional affiliations.

Received: 18 October 2017 Accepted: 12 January 2018 Published online: 02 February 2018

\section{References}

1. Bohr, H: Zur Theorie der fastperiodischen Funktionen I. Acta Math. 45, 29-127 (1925)

2. Bohr, H: Zur Theorie der fastperiodischen Funktionen II. Acta Math. 46, 101-204 (1925)

3. Bohr, H: Zur Theorie der fastperiodischen Funktionen III. Acta Math. 47, 237-281 (1926)

4. Amerio, L, Prouse, G: Almost-Periodic Functions and Functional Equations. Springer, New York (1971)

5. Levitan, BM: Almost Periodic Functions. Gos. Izdat. Tekhn-Teor. Lit., Moscow (1953) (in Russian)

6. Besicovitch, AS: Almost Periodic Functions. Dover, New York (1954)

7. Bochner, S: Curvature and Betti numbers in real and complex vector bundles. Rend. Semin. Mat. (Torino) 15, 225-253 (1955-1956)

8. Corduneanu, C: Almost Periodic Functions. Wiley, New York (1968)

9. Favard, J: Leçons sur les fonctions presque périodiques. Gauthier-Villars, Paris (1933) (in French)

10. Fink, AM: Almost Periodic Differential Equations. Lecture Notes in Mathematics, vol. 377. Springer, Berlin (1974)

11. Bochner, S: A new approach to almost periodicity. Proc. Natl. Acad. Sci. USA 48, 2039-2043 (1962)

12. Landau, L, Lifschitz, E: Cours de Physique théorique, tome 6, 2nd edn. Mir, Moscow (1989) (in French)

13. Allais, M: Sur la distribution normale des valeurs à des instants régulièrement espacés d'une somme des sinusoïdes. In: Comptes Rendus de l'Académie des Sciences de Paris, tome 296, Série I, pp. 829-832 (1983) (in French)

14. Lassoued, D: Fonctions presque-périodiques et équations différentielles. Ph.D. Dissertation, Université Paris I, Panthéon-Sorbonne, Laboratoire SAMM (December 2013) (in French)

15. Levitan, BM, Zhikov, W: Almost Periodic Functions and Differential Equations. Cambridge University Press, London (1982)

16. Corduneanu, C: Almost Periodic Functions, 2nd edn. Chelsea, New York (1989)

17. Diagana, T: Almost Automorphic Type and Almost Periodic Type Functions in Abstract Spaces. Springer, New York (2013)

18. N'Guérékata, GM: Almost Automorphic and Almost Periodic Functions in Abstract Spaces. Kluwer Academic, Dordrecht (2001)

19. N'Guérékata, GM: Topics in Almost Automorphy. Springer, New York (2005)

20. Zaidman, S: Almost-Periodic Functions in Abstract Spaces. Pitman Research Notes in Mathematics, vol. 126. Pitman, Boston (1985)

21. Arendt, W, Batty, CJK, Hieber, M, Neubrander, F: Vector-Valued Laplace Transforms and Cauchy Problems. Birkhäuser, Basel (2001)

22. Bart, $\mathrm{H}$, Goldberg, S: Characterizations of almost periodic strongly continuous groups and semigroups. Math. Ann. 236, 105-116 (1978)

23. Corduneanu, C: Almost Periodic Oscillations and Waves. Springer, Berlin (2010)

24. Batty, CJK, van Neerven, J, Räbiger, F: Tauberian theorems and stability of solutions of the Cauchy problem. Trans. Am. Math. Soc. 350, 2087-2103 (1998)

25. Cheban, DN: Asymptotically Almost Periodic Solutions of Differential Equations. Hindawi Publishing Corporation New York (2009)

26. Deleeuw, K, Glicksberg, I: Applications of almost periodic compactifications. Acta Math. 105, 63-97 (1961)

27. Ruess, WM, Summers, WH: Compactness in spaces of vector valued continuous functions and asymptotic almost periodicity. Math. Nachr. 135, 7-33 (1988)

28. Sathiyanathan, K, Nandha Gopal, T: Existence of asymptotically almost periodic solutions of integrodifferential equations. Appl. Math. Comput. Intell. 2, 205-216 (2013)

29. Xie, L, Li, M, Huang, F: Asymptotic almost periodicity of C-semigroups. Int. J. Math. Math. Sci. 2, 65-73 (2003)

30. Zhang, C: Vector-valued pseudo almost periodic functions. Czechoslov. Math. J. 47, 385-394 (1997)

31. Ruess, WM, Summers, WH: Asymptotic almost periodicity and motions of semigroups of operators. Linear Algebra Appl. 84, 335-351 (1986)

32. Ruess, WM, Summers, WH: Integration of asymptotically almost periodic functions and weak asymptotic almost periodicity. Diss. Math. 279, 1-35 (1989)

33. Ruess, WM, Vũ, QP: Asymptotically almost periodic solutions of evolution equations in Banach spaces. J. Differ. Equ. 122, 282-301 (1995)

34. Zhang, C: Ergodicity and asymptotically almost periodic solutions of some differential equations. Int. J. Math. Math. Sci. 25(12), 787-800 (2001)

35. Long, W, Ding, H-S: Composition theorems of Stepanov almost periodic functions and Stepanov-like pseudo-almost periodic functions. Adv. Differ. Equ. 2011, Article ID 654695 (2011). https://doi.org/10.1155/2011/654695

36. Henríquez, HR: On Stepanov-almost periodic semigroups and cosine functions of operators. J. Math. Anal. Appl. 146, 420-433 (1990)

37. Andres, J, Bersani, AM, Grande, RF: Hierarchy of almost-periodic function spaces. Rend. Mat. Appl. (7) 26, 121-188 (2006) 
38. Andres, J, Bersani, AM, Leśniak, K: On some almost-periodicity problems in various metrics. Acta Appl. Math. 65, 35-57 (2001)

39. Kovanko, AS: Sur la compacité des systèmes de fonctions presque périodiques généralisées de H. Weyl. C. R. (Dokl.) Acad. Sci. URSS 43, 275-276 (1944) (in French)

40. Danilov, LI, Kudryavtsev, LD, Levitan, BM: Elements of the Theory of Functions. Pergamon, Oxford (1966)

41. Diagana, T, Nelson, V, N'Guérékata, GM: Stepanov-like $C^{(n)}$-pseudo almost automorphy and applications to some nonautonomous higher-order differential equations. Opusc. Math. 32, 455-471 (2012)

Submit your manuscript to a SpringerOpen ${ }^{\circ}$ journal and benefit from:

- Convenient online submission

$\checkmark$ Rigorous peer review

- Open access: articles freely available online

- High visibility within the field

Retaining the copyright to your article

Submit your next manuscript at $\boldsymbol{\nabla}$ springeropen.com 